\title{
Linear independence of the T-spline blending functions associated with some particular T-meshes
}

\author{
A. Buffa ${ }^{a}$, D. Cho ${ }^{a}$, G. Sangalli ${ }^{b}$ \\ ${ }^{a}$ Istituto di Matematica Applicata e Tecnologie Informatiche del CNR, Via Ferrata, 1; 27100 Pavia; Italy. \\ ${ }^{b}$ Dipartimento di Matematica, Università di Pavia; Via Ferrata, 1; 27100 Pavia; Italy.
}

\begin{abstract}
Based on the local refinement algorithm addressed in [18], we analyze the linear independence of the bi-cubic T-spline blending functions corresponding to particular T-meshes.
\end{abstract}

Key words:

Isogeometric analysis, T-splines, Linear independence

\section{Introduction}

Recently, the so-called Isogeometric Analysis was introduced in Hughes, Cottrell and Bazilevs [16], which is based on developments in the modeling of complex geometries. The new methodology based on nonuniform rational B-splines (NURBS) has been extensively studied in e.g., $[2,10,12,14,8]$ and applied in fields as diverse as fluid dynamics in $[5,6$, $4,7,15]$, structural mechanics in $[1,11,13,20,21]$ and electromagnetics in the a pioneering paper [9].

Important among advantages that NURBS-based isogeometric analysis possesses are to fix exact geometries at the coarsest level of discretization and eliminate geometrical errors. Variational formulations of the partial differential equations (PDEs) are also derived from NURBS-based isogeometric analysis. Its solution space is represented in terms of the same basis functions as the geometry, which have nice properties such as partition of unity, pointwise nonnegativity, linear independence, compact support and control of continuity. A significant disadvantage of NURBS is that they are based on a tensor product structure and, as a consequence, knot insertion is a global operation. This means that NURBS models may have a large number of superfluous control points (knots) at each step of refinement.

A potential extension of isogeometric concept is T-splines that were introduced in Sederberg and his collaborators $[18,19]$. T-splines not only retain precise geometric representation of NURBS, but also allow for the local refinement. Isogeometric analysis using T-splines has been further explored in $[3,12]$.

In the framework of isogeometric analysis using T-splines, several theoretical questions about T-splines have been still open for the last five years. Most fundamental among the open problems is the linear independence of the associated T-spline blending functions with

Email addresses: annalisa.buffa@imati.cnr.it (A. Buffa), durkbin@imati.cnr.it (D. Cho), giancarlo.sangalli@unipv.it (G. Sangalli) 
T-meshes. Linear independence is not guaranteed on a generic T-mesh: we show in this paper examples of T-meshes with repeated knots that induce linear dependent blending functions. However, we also prove the linear independence of the set of blending functions associated with some particular T-meshes of practical interest, such as nonmatching ones, and the ones associated with geometric refinement towards edges, layers or corners. This is a first step in the study of T-meshes, the full characterization of linear independence being still open.

The paper is organized as follows. In Section 2, we introduce T-meshes and discuss their corresponding bi-cubic T-spline blending functions. In Section 3, we show counterexamples to linear independence, and study the linear independence of T-splines over particular Tmeshes. In Section 4, we apply the results described in Section 3 to present several examples of linearly independent T-splines. Finally, we end with the concluding remarks in Section 5.

\section{Preliminary}

\subsection{Univariate splines}

We briefly introduce univariate B-splines with the aim of fixing our notation, and recalling a few results that we will need in the next sections. We only restrict to the case of cubic splines.

Given the integer $k>0$, let a knot vector $\Xi=\left\{\xi_{-1}, \xi_{0}, \xi_{1}, \ldots \xi_{k}, \xi_{k+1}, \xi_{k+2}\right\}$ be given, with ordered knots $\xi_{i} \leq \xi_{i+1}$. The knot vector $\Xi$ is said to be open if the first 4 and last 4 knots are repeated, that is $\xi_{-1}=\xi_{0}=\xi_{1}=\xi_{2}$ and $\xi_{k-1}=\xi_{k}=\xi_{k+1}=\xi_{k+2}$. All the other knots have multiplicity less than or equal to 4 . For $1 \leq i \leq k$, we denote by

$$
x \mapsto N\left[\xi_{i-2}, \xi_{i-1}, \xi_{i}, \xi_{i+1}, \xi_{i+2}\right](x),
$$

the cubic (univariate) B-spline function associated with knots $\left\{\xi_{i-2}, \xi_{i-1}, \xi_{i}, \xi_{i+1}, \xi_{i+2}\right\}$. When a knot has multiplicity $r, r=2,3,4$, the B-splines are at most $C^{3-r}$ at that knot, in general. Following [17, Theorem 4.41], we define suitable functionals which are dual to the B-splines basis. Let

$$
G_{j}(x)=g\left(\frac{2 x-\xi_{j-2}-\xi_{j+2}}{\xi_{j+2}-\xi_{j-2}}\right),
$$

where $g$ is the transition function defined in [17, Theorem 4.37] and

$$
\phi_{j}(x)=\frac{\left(x-\xi_{j-1}\right) \cdots\left(x-\xi_{j+1}\right)}{6},
$$

We define the Schumaker's functional $\lambda^{j}$ associated with $\Xi$ as follows: for $j=1, \ldots, n$,

$$
\lambda^{j}(f):=\int_{\xi_{j-2}}^{\xi_{j+2}} f(x) D^{4} \psi_{j}(x) d x, \quad \forall f \in L^{2}[0,1],
$$

where $\psi_{j}(x)=G_{j}(x) \phi_{j}(x)$. Note that $\lambda^{j}$ is associated with $\operatorname{knots}\left\{\xi_{j-2}, \xi_{j-1}, \xi_{j}, \xi_{j+1}, \xi_{j+2}\right\}$ and depends then on the chosen knot vector $\Xi$. The functional $\lambda^{j}$ are constructed to verify the following property:

$$
\lambda^{j}\left(N\left[\xi_{i-2}, \xi_{i-1}, \xi_{i}, \xi_{i+1}, \xi_{i+2}\right](s)\right)=\delta_{i j}, \quad 1 \leq i, j \leq n,
$$

where $\delta_{i j}$ is the Kronecker delta. 


\subsection{T-mesh and definition of T-splines}

We start this section with an abstract definition of (two-dimensional) T-mesh. Let $\mathcal{I}_{\mathfrak{i}}=$ $\{1,2, \ldots, n\}$ and $\mathcal{I}_{\mathfrak{j}}=\{1,2, \ldots, m\}$ be two sets of indexes, let $\mathfrak{A} \subseteq \mathcal{I}_{\mathfrak{i}} \times \mathcal{I}_{\mathfrak{j}}$, denoting a set of anchors, and let $\mathfrak{E}$ be a set of horizontal or vertical closed edges between anchors, that is

$$
\mathfrak{E} \subseteq\left\{\left[\left(i_{1}, j_{1}\right),\left(i_{2}, j_{2}\right)\right] \text { such that }\left(i_{1}, j_{1}\right) \neq\left(i_{2}, j_{2}\right) \in \mathfrak{A}, \text { with } i_{1}=i_{2} \text { or } j_{1}=j_{2}\right\} \text {; }
$$

let $\Xi_{\mathfrak{s}}=\left\{s_{-1}, \ldots, s_{n+2}\right\}$ and $\Xi_{\mathfrak{t}}=\left\{t_{-1}, \ldots, t_{m+2}\right\}$ be open knot vectors on the interval [0,1], that is, $\Xi_{\mathfrak{s}}: 0=s_{-1}=s_{0}=s_{1}=s_{2}<s_{3} \leq s_{4} \leq \ldots \leq s_{n-3} \leq s_{n-2}<s_{n-1}=s_{n}=$ $s_{n+1}=s_{n+2}=1$ and analogously $\Xi_{\mathfrak{t}}: 0=t_{-1}=t_{0}=t_{1}=t_{2}<t_{3} \leq t_{4} \leq \ldots \leq t_{m-3} \leq$ $t_{m-2}<t_{m-1}=t_{m}=t_{m+1}=t_{m+2}=1$, with all internal knots having multiplicity less or equal than 3. A T-mesh $\mathfrak{M}=\mathfrak{M}\left(\mathcal{I}_{\mathfrak{i}}, \mathcal{I}_{\mathfrak{j}}, \mathfrak{A}, \mathfrak{E}, \Xi_{\mathfrak{s}}, \Xi_{\mathfrak{t}}\right)$ is defined by the previous objects, when the following condition are fulfilled:

1. $\forall e_{1}, e_{2} \in \mathfrak{E}, e_{1} \cap e_{2} \in \mathfrak{A}$, that is, there is an anchor on each edge intersection, and edges do not overlap,

2. $\forall a \in \mathfrak{A}, \exists e \in \mathfrak{E} \mid a \in e$, that is, there are no isolated anchors.

The condition 1 above slightly extends the previous definition of T-meshes in [3, 18, 19], where only T-junctions, L-junctions or four-edges junctions are allowed.

A graphical representation of the T-mesh $\mathfrak{M}$ is obtained drawing anchors and edges in the so called index space, or in the index/parametric space or in the parametric space, as is shown in Figure 1. In the index space, $i$ represents the horizontal index and $j$ the vertical one. In the parametric space, the horizontal and vertical variables are $s$ and $t$ respectively. Once a T-mesh $\mathfrak{M}$ is given in the index space, the corresponding T-mesh on the parametric space is obtained by mapping indexes on the corresponding knot vectors, namely, $i \mapsto s_{i}, j \mapsto t_{j}$. For a fixed $\overline{\mathrm{j}} \in \mathcal{I}_{\mathfrak{j}}$, we define

$$
\mathcal{I}_{\mathfrak{i}}^{\bar{\jmath}}:=\{i:(i, \overline{\mathfrak{j}}) \in \mathfrak{A} \text { or there exists a vertical edge } e \in \mathfrak{E} \text { such that }(i, \overline{\mathrm{j}}) \in e\},
$$

and $\Xi_{\mathfrak{s}}^{\bar{j}}$ as the vector of the corresponding $s$-knots to the indexes in $\mathcal{I}_{\mathfrak{i}}^{\bar{\jmath}}$. Similarly, for fixed $\overline{1} \in \mathcal{I}_{\mathfrak{i}}$, we define

$$
\mathcal{I}_{\mathfrak{j}}^{\overline{1}}:=\{j:(\overline{1}, j) \in \mathfrak{A} \text { or there exists a horizontal edge } e \in \mathfrak{E} \text { such that }(\overline{1}, j) \in e\},
$$

and $\Xi_{\mathfrak{t}}^{\overline{1}}$ as the vector of the corresponding $t$-knots to the indexes in $\mathcal{I}_{\mathfrak{j}}^{\overline{1}}$. For example, let us consider the T-mesh in Figure 1. Here $n=6, m=7, \Xi_{\mathfrak{s}}=\left\{s_{-1}, s_{0}, s_{1}, s_{2}, s_{3}, s_{4}, s_{5}, s_{6}, s_{7}, s_{8}\right\}$ and $\Xi_{\mathfrak{t}}=\left\{t_{-1}, t_{0}, t_{1}, t_{2}, t_{3}, t_{4}, t_{5}, t_{6}, t_{7}, t_{8}, t_{9}\right\}$ are the global knot vectors of T-mesh $\mathfrak{M}$ in Figure 1. Then, we have the following index/knot association:

$$
\begin{aligned}
& \mathcal{I}_{\mathfrak{i}}=\{1,2, \ldots, 6\} \rightarrow\left\{s_{1}, s_{2}, \ldots, s_{6}\right\}=\{0,0,1 / 3,2 / 3,1,1\} \\
& \mathcal{I}_{\mathfrak{j}}=\{1,2, \ldots, 7\} \rightarrow\left\{t_{1}, t_{2}, \ldots, t_{7}\right\}=\{0,0,1 / 4,1 / 2,3 / 4,1\} .
\end{aligned}
$$

Therefore, for this example, we get

$$
\begin{aligned}
& \mathcal{I}_{\mathfrak{i}}^{3}=\{1,2,3,4,5,6\}, \quad \Xi_{\mathfrak{s}}^{3}=\{0,0,1 / 3,2 / 3,1,1\} \\
& \mathcal{I}_{\mathfrak{i}}^{5}=\{1,2,3,5,6\}, \quad \Xi_{\mathfrak{s}}^{5}=\{0,0,1 / 3,1,1\} \\
& \mathcal{I}_{\mathfrak{j}}^{4}=\{1,2,3,4,6,7\}, \quad \Xi_{\mathfrak{t}}^{4}=\{0,0,1 / 4,1 / 2,1,1\} .
\end{aligned}
$$




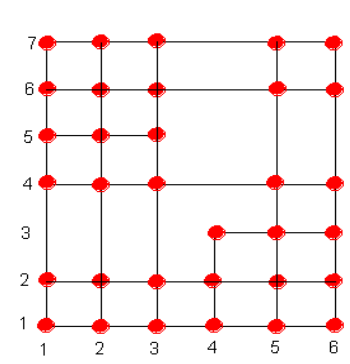

(a) Index space

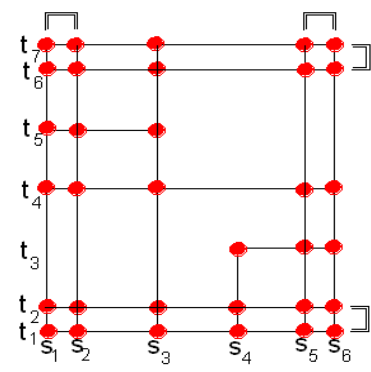

(b) Index/parametric space

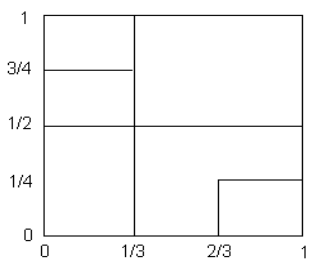

(c) Parametric space

Figure 1: Repeated lines in (b) are expanded to show the relation between index and parametric spaces.

Also, we have an anchor as each vertex of the T-mesh in the index space. Each anchor is designated by a circle in Figure 1(a). Though it is not mandatory, we always place two lines of anchors at the boundary of the index space, which correspond to knots with multiplicity 4. On the one hand, the mesh on the parametric space is the one we map exactly onto the computational domain, on the other hand it does not bring all the information as the corresponding T-mesh on the index space in case of knot repetition. In Figure 1(b), we show the index/parametric representation of the T-mesh, in which we encode all the information. The vertical and horizontal lines that are drawn closer and marked by parallel brackets correspond to knot repetition.

Let $\mathfrak{M}$ be a T-mesh. $\mathfrak{M}$ is used to define a set of B-spline functions, called T-spline blending function, on the parametric domain $[0,1]^{2}$. We attach a T-spline blending function to each anchor of $\mathfrak{M}$. Given $(\overline{1}, \overline{\mathrm{j}}) \in \mathfrak{A}$, the corresponding blending function is constructed as the product of a spline in the $s$ direction, $s \mapsto N_{\mathfrak{s}}^{(\overline{1}, \bar{j})}(s)$ and one in the $t$ direction, $t \mapsto N_{\mathfrak{t}}^{(\overline{1}, \bar{j})}(t)$. $N_{\mathfrak{s}}^{(\overline{1}, \overline{\mathrm{j}})}$ has $s_{\overline{\mathrm{l}}}$ as a center knot and is constructed on the knot vector $\Xi_{\mathfrak{s}}^{\overline{\mathrm{j}}}$, while $N_{\mathfrak{t}}^{(\overline{1}, \overline{\mathrm{j}})}$ has $t_{\overline{\mathrm{j}}}$ as a center knot and is constructed on the knot vector $\Xi_{\mathrm{t}}^{\overline{1}}$. Finally, the B-spline attached to $(\overline{1}, \bar{\jmath}) \in \mathfrak{A}$ is:

$$
(s, t) \mapsto B^{(\overline{1}, \bar{\jmath})}(s, t)=N_{\mathfrak{s}}^{(\overline{1}, \bar{j})}(s) N_{\mathfrak{t}}^{(\overline{1}, \bar{j})}(t) .
$$

As an example, we construct the blending function associated with the anchor $(3,4)$ of the T-mesh in Figure 1(a). From the knot vectors:

$\Xi_{\mathfrak{s}}^{4}=\left\{s_{1}, s_{2}, s_{3}, s_{5}, s_{6}\right\}=\{0,0,1 / 3,1,1\} \quad$ and $\quad \Xi_{\mathfrak{t}}^{3}=\left\{t_{1}, t_{2}, t_{4}, t_{5}, t_{6}, t_{7}\right\}=\{0,0,1 / 2,3 / 4,1,1\}$

and we extract the local knot vectors

$$
\left\{s_{1}, s_{2}, s_{3}, s_{5}, s_{6}\right\}=\{0,0,1 / 3,1,1\} \text { and }\left\{t_{1}, t_{2}, t_{4}, t_{5}, t_{6}\right\}=\{0,0,1 / 2,3 / 4,1\},
$$

and construct the T-spline

$$
\begin{aligned}
B^{(3,4)}(s, t) & :=N[0,0,1 / 3,1,1](s) N[0,0,1 / 2,3 / 4,1](t) \\
& :=N_{\mathfrak{s}}^{(3,4)}(s) N_{\mathfrak{t}}^{(3,4)}(t) .
\end{aligned}
$$

Accordingly, each anchor and its corresponding bi-cubic T-spline blending function are in one-to-one correspondence. With the given T-mesh $\mathfrak{M}$, the associated bi-cubic T-spline blending functions span a function space, denoted by

$$
S:=\operatorname{span}\left\{B^{(i, j)}(s, t): B^{(i, j)}(s, t)=N_{\mathfrak{s}}^{(i, j)}(s) N_{\mathfrak{t}}^{(i, j)}(t),(i, j) \in \mathfrak{A}\right\},
$$


where $\mathfrak{A}$ is the set of anchors on the T-mesh $\mathfrak{M}$. With an abuse of notation, we say that

$\mathfrak{M}$ is a linearly independent T-mesh $\Leftrightarrow \quad B^{(i, j)},(i, j) \in \mathfrak{A}$ are linearly independent

In this case, the dimension of $S$ is $\# \mathfrak{A}$, where $\#$ denotes the cardinality. We also refer to $[3,12,18,19]$ for T-meshes and their corresponding T-spline blending functions.

Remark 2.1. According with [18], one can classify T-splines spaces in:

- standard when

$$
\sum_{(i, j) \in \mathfrak{A}} B^{(i, j)}(s, t)=1
$$

- semi-standard when $1 \in S$ without (5),

- non-standard when $1 \notin S$.

In the last case, one can consider the space of rational T-splines as

$$
S_{R}:=\operatorname{span}\left\{\frac{B^{(i, j)}(s, t)}{\sum_{(k, \ell) \in \mathfrak{A}} w^{(k, \ell)} B^{(k, \ell)}(s, t)},(i, j) \in \mathfrak{A}\right\},
$$

where $w^{(k, \ell)}$ are chosen strictly positive weights. Notice that

$$
\begin{aligned}
& B^{(i, j)}(s, t),(i, j) \in \mathfrak{A} \text { are linearly independent } \\
& \Leftrightarrow \quad \frac{B^{(i, j)}(s, t)}{\sum_{(k, \ell) \in \mathfrak{A}} w^{(k, \ell)} B^{(k, \ell)}(s, t)},(i, j) \in \mathfrak{A} \text { are linearly independent. }
\end{aligned}
$$

For this reason, we will consider only the space $S$ in (3) in this paper.

\section{Linear Independence of T-splines}

First of all, a question arises if any T-mesh is always linearly independent according to (4). The answer is negative, as it is shown in the example represented in Figure 2. The associated T-splines with anchors $(3,4),(4,5)$ and $(5,5)$ shown in the index space

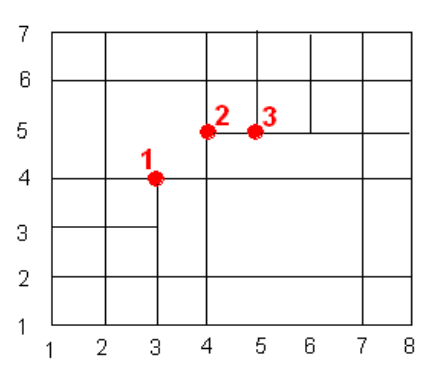

(a) Index space

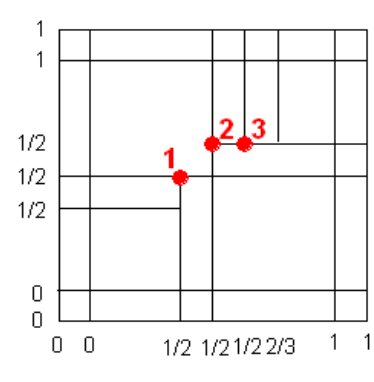

(b) Indexiparametric space

Figure 2: A counterexample of linearly independent T-splines. 
(Figure $2(\mathrm{a}))$, which correspond to (repeated) image $\left(s_{3}, t_{4}\right)=\left(s_{4}, t_{5}\right)=\left(s_{5}, t_{5}\right)=(1 / 2,1 / 2)$ in the index/parametric space (Figure 2(b)), are obtained as follows:

$$
\begin{gathered}
\Theta_{1}(s, t)=N[0,0,1 / 2,1 / 2,1](s) N[0,1 / 2,1 / 2,1,1](t), \\
\Theta_{2}(s, t)=N[0,0,1 / 2,1 / 2,2 / 3](s) N[0,1 / 2,1 / 2,1,1](t),
\end{gathered}
$$

and

$$
\Theta_{3}(s, t)=N[0,1 / 2,1 / 2,2 / 3,1](s) N[0,1 / 2,1 / 2,1,1](t),
$$

respectively. By the rules of knot insertion, $\Theta_{1}=\Theta_{2}+\frac{1}{3} \Theta_{3}$, and the bi-cubic T-spline blending functions associated with the T-mesh illustrated in Figure 2(a) are linearly dependent. This means that T-splines do not always form a set of linearly independent functions, or, equivalently, that not all T-meshes are linearly independent in the sense of (4).

In the remaining of this section, we analyze the linear independence of the bi-cubic Tspline blending functions associated with T-meshes of practical interest and that are obtained by one (or several) application(s) of the local refinement algorithm defined in [18].

We introduce some notation that will be used in what follows. Let a starting T-mesh $\mathfrak{M}_{0}$ be given. After we add a (or several) anchor(s) to T-mesh $\mathfrak{M}_{0}$, we obtain a new T-mesh $\mathfrak{M}_{1}$. This process may be repeated several times if necessary: $\mathfrak{M}_{0}, \mathfrak{M}_{1}, \ldots$, and $\mathfrak{M}_{\ell}$. Without loss of generality and in order to have an adequate notation, we suppose that all the T-meshes have in common the index sets $\mathcal{I}_{\mathfrak{i}}$ and $\mathcal{I}_{\mathfrak{j}}$ and the knot vectors $\Xi_{\mathfrak{s}}$ and $\Xi_{\mathfrak{t}}$. Each T-mesh $\mathfrak{M}_{k}$ is characterized by its set of anchors $\mathfrak{A}_{k}$ and the set of edges $\mathfrak{E}_{k}$. All the quantities in the previous section will be indexed by the T-mesh index $k$ to stress that they are referred to the T-mesh $\mathfrak{M}_{k}$. For instance, fix a $\bar{\jmath} \in \mathcal{I}_{\mathfrak{j}}$, we define $\mathcal{I}_{\mathfrak{i}, k}^{\bar{j}}$ and $\Xi_{\mathfrak{s}, k}^{\bar{j}}$ as in the previous section. Also, the sequence of the spaces spanned by the bicubic T-splines inferred from T-meshes $\mathfrak{M}_{0}, \mathfrak{M}_{1}, \ldots$, and $\mathfrak{M}_{\ell}$ is

$$
\begin{aligned}
S_{0} & :=\operatorname{span}\left\{B_{0}^{(i, j)}(s, t): B_{0}^{(i, j)}(s, t)=N_{\mathfrak{s}, 0}^{(i, j)}(s) N_{\mathfrak{t}, 0}^{(i, j)}(t),(i, j) \in \mathfrak{A}_{0}\right\} \\
S_{1} & :=\operatorname{span}\left\{B_{1}^{(i, j)}(s, t): B_{1}^{(i, j)}(s, t)=N_{\mathfrak{s}, 1}^{(i, j)}(s) N_{\mathfrak{t}, 1}^{(i, j)}(t),(i, j) \in \mathfrak{A}_{1}\right\} \\
& \ldots \\
S_{\ell} & :=\operatorname{span}\left\{B_{\ell}^{(i, j)}(s, t): B_{\ell}^{(i, j)}(s, t)=N_{\mathfrak{s}, \ell}^{(i, j)}(s) N_{\mathfrak{t}, \ell}^{(i, j)}(t),(i, j) \in \mathfrak{A}_{\ell}\right\}
\end{aligned}
$$

respectively.

Within this framework, we also assume that the T-mesh $\mathfrak{M}_{k+1}$ is obtained from $\mathfrak{M}_{k}$ by inserting new anchors, that is,

$$
\mathfrak{A}_{0} \subset \mathfrak{A}_{1} \subset \cdots \subset \mathfrak{A}_{\ell}
$$

Observe that (7) does not imply that $S_{0} \subset S_{1} \subset \cdots \subset S_{\ell}$. We say that

$$
\mathfrak{M}_{k+1} \text { is a refinement of } \mathfrak{M}_{k} \Leftrightarrow \quad S_{k} \subset S_{k+1} \text {; }
$$

this is guaranteed by the local refinement algorithm described in [18].

The following holds:

Proposition 3.1. Let $\mathfrak{M}_{0}$ be a linearly independent $T$-mesh, and $\mathfrak{M}_{1}$ a refinement of $\mathfrak{M}_{0}$. If $\left\{B_{1}^{(i, j)}(s, t):(i, j) \in \mathfrak{A}_{1} \backslash \mathfrak{A}_{0}\right\}$ is a linearly independent set and the sum of $S_{0}$ with 
$\operatorname{span}\left\{B_{1}^{(i, j)}(s, t):(i, j) \in \mathfrak{A}_{1} \backslash \mathfrak{A}_{0}\right\}$ is direct, then $\mathfrak{M}_{1}$ is linearly independent and in particular it holds:

$$
S_{0} \oplus \operatorname{span}\left\{B_{1}^{(i, j)}(s, t):(i, j) \in \mathfrak{A}_{1} \backslash \mathfrak{A}_{0}\right\}=S_{1}
$$

ProOF. It follows from the local refinement algorithm that both the sets $\left\{B_{0}^{(i, j)},(i, j) \in \mathfrak{A}_{0}\right\}$ and $\left\{B_{1}^{(i, j)},(i, j) \in \mathfrak{A}_{1} \backslash \mathfrak{A}_{0}\right\}$ are contained in $S_{1}$. Then, they span a subset of $S_{1}$. However,

$$
\operatorname{dimension}\left(S_{0} \oplus \operatorname{span}\left\{B_{1}^{(i, j)}(s, t):(i, j) \in \mathfrak{A}_{1} \backslash \mathfrak{A}_{0}\right\}\right)=\# \mathfrak{A}_{0}+\#\left(\mathfrak{A}_{1} \backslash \mathfrak{A}_{0}\right)=\# \mathfrak{A}_{1} ;
$$

thus $S_{0} \oplus \operatorname{span}\left\{B_{1}^{(i, j)}(s, t):(i, j) \in \mathfrak{A}_{1} \backslash \mathfrak{A}_{0}\right\}$ and $S_{1}$ coincide and the proof is complete.

The statement in Proposition 3.1 is easy, but provide us with a strategy to prove linear independence for T-splines blending functions. That is, given a linearly independent T-mesh $\mathfrak{M}_{0}$ and its refinement $\mathfrak{M}_{1}$, we have to show the following:

i) the functions we add, namely $\left\{B_{1}^{(i, j)}(s, t),(i, j) \in \mathfrak{A}_{1} \backslash \mathfrak{A}_{0}\right\}$ are linearly independent;

ii) the sum in the left side of (9) is direct.

The following lemmas are based on this simple idea and play a crucial role in the proof of linear independence of the resulting T-splines.

Lemma 3.2. Consider a linearly independent T-mesh $\mathfrak{M}_{0}$, and a T-mesh $\mathfrak{M}_{1}$ obtained by adding anchors (to $\mathfrak{M}_{0}$ ) with the property that there exists a unique $\overline{\mathrm{j}} \in \mathcal{I}_{\mathfrak{j}}$ such that

$$
\mathfrak{A}_{1} \backslash \mathfrak{A}_{0}=\left\{(i, \overline{\mathrm{j}}), i \in \mathcal{I}_{\mathfrak{i}}\right\} \cap \mathfrak{A}_{1},
$$

and such that

$$
\forall(i, j) \in \mathfrak{A}_{0}, t_{j} \neq t_{\overline{\mathrm{J}}} .
$$

Then the functions $\left\{B_{1}^{(i, \bar{\jmath})},(i, \bar{\jmath}) \in \mathfrak{A}_{1} \backslash \mathfrak{A}_{0}\right\}$ are linearly independent, and the following sum is direct

$$
S_{0} \oplus \operatorname{span}\left\{B_{1}^{(i, \bar{\jmath})}(s, t), \quad(i, \bar{\jmath}) \in \mathfrak{A}_{1} \backslash \mathfrak{A}_{0}\right\}
$$

The condition (10) is saying that we add anchors living on a new horizontal line in the index space, and the condition (11) says that this line is mapped onto a new line in the parametric space. Before proving this Lemma, we notice that $\mathfrak{M}_{1}$ may not be a refinement of $\mathfrak{M}_{0}$. Examples of those $\mathfrak{M}_{1}$ are presented in Figure 3 . Indeed, the local refinement algorithm[18] would ask for additional insertion. Moreover, it should be remarked that there are no restrictions in the position of new anchors in the horizontal direction.

We are now in position to prove Lemma 3.2.

Proof of Lemma 3.2. Suppose that

$$
\sum_{(i, j) \in \mathfrak{A}_{0}} \alpha^{(i, j)} B_{0}^{(i, j)}(s, t)+\sum_{(i, \bar{\jmath}) \in \mathfrak{A}_{1} \backslash \mathfrak{A}_{0}} \beta^{(i, \bar{\jmath})} B_{1}^{(i, \bar{\jmath})}(s, t)=0,
$$




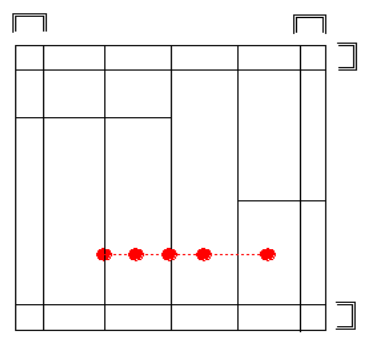

(a)

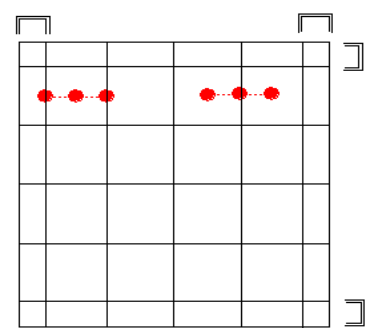

(b)

Figure 3: Dotted lines denote newly added lines on T-mesh $\mathfrak{M}_{0}$ in index/parametric spaces. New anchors are identified with circles. Parallel brackets denote repeated lines as in Figure 1.

with constants $\alpha^{(i, j)},(i, j) \in \mathfrak{A}_{0}, \beta^{(i, \bar{\jmath})},(i, \bar{\jmath}) \in \mathfrak{A}_{1} \backslash \mathfrak{A}_{0}$. Considering the knot vector $\Xi_{\mathfrak{s}, 1}^{\bar{j}}$ we can define, following Section 2.1, a set of dual functionals $\lambda_{\mathfrak{s}}^{(i, \bar{\jmath})}$ for the univariate splines $N_{\mathfrak{s}, 1}^{(i, \bar{\jmath})}$. We then define a tensor product homomorphism $\Phi_{\mathfrak{s}}^{(i, \bar{\jmath})}:=\lambda_{\mathfrak{s}}^{(i, \bar{\jmath})} \otimes$ id from $L^{2}[0,1] \otimes L^{2}[0,1]$ into $\mathbb{R} \otimes L^{2}[0,1]$ by

$$
\Phi_{\mathfrak{s}}^{(i, \bar{\jmath})}(f \otimes g)(s, t)=\left(\lambda_{\mathfrak{s}}^{(i, \bar{\jmath})} \otimes \mathrm{id}\right)(f \otimes g)(s, t)=\lambda_{\mathfrak{s}}^{(i, \bar{\jmath})}(f(s)) g(t),
$$

where id denotes the identity homomorphism. Taking $\Phi_{\mathfrak{s}}^{(i, \bar{\jmath})}$ on both sides of (13) leads to

$$
\begin{aligned}
& \Phi_{\mathfrak{s}}^{(i, \bar{\jmath})}\left(\sum_{(i, j) \in \mathfrak{A}_{0}} \alpha^{(i, j)} B_{0}^{(i, j)}(s, t)+\sum_{(i, \bar{\jmath}) \in \mathfrak{A}_{1} \backslash \mathfrak{A}_{0}} \beta^{(i, \bar{\jmath})} B_{1}^{(i, \bar{\jmath})}(s, t)\right)=0 \\
& \Phi_{\mathfrak{s}}^{(i, \bar{\jmath})}\left(\sum_{(i, j) \in \mathfrak{A}_{0}} \alpha^{(i, j)} B_{0}^{(i, j)}(s, t)\right)=-\beta^{(i, \bar{\jmath})} N_{\mathfrak{t}, 1}^{(i, \bar{\jmath})}(t) .
\end{aligned}
$$

Observe that $\Phi_{\mathfrak{s}}^{(i, \bar{\jmath})}\left(\sum_{(i, j) \in \mathfrak{A}_{0}} \alpha^{(i, j)} B_{0}^{(i, j)}(s, t)\right)$ is $C^{\infty}$ at $t=t_{\overline{\mathrm{J}}}$ and $N_{\mathfrak{t}, 1}^{(i, \overline{\mathrm{J}})}(t)$ is not $C^{\infty}$ at that point. Therefore for any $(i, \overline{\mathrm{J}}) \in \mathfrak{A}_{1} \backslash \mathfrak{A}_{0}, \beta^{(i, \overline{\mathrm{j}})}$ is zero. So, the functions $\left\{B_{1}^{(i, \overline{\mathrm{J}})}(s, t),(i, \overline{\mathrm{J}}) \in\right.$ $\left.\mathfrak{A}_{1} \backslash \mathfrak{A}_{0}\right\}$ are linearly independent. Also, $\alpha^{(i, j)}$ is zero for any $(i, j) \in \mathfrak{A}_{0}$ since $\left\{B_{0}^{(i, j)}\right\}_{(i, j) \in \mathfrak{A}_{0}}$ are linearly independent by assumption. Consequently, the sum in (12) is direct.

Remark 3.3. By exchanging vertical with horizontal lines in the statement of Lemma 3.2, an equivalent result holds true when adding new anchors with the property that there exists a unique $\overline{1} \in \mathcal{I}_{\mathfrak{i}}$ such that

$$
\mathfrak{A}_{1} \backslash \mathfrak{A}_{0}=\left\{(\overline{1}, j), j \in \mathcal{I}_{\mathfrak{j}}\right\} \cap \mathfrak{A}_{1},
$$

and such that

$$
\forall(i, j) \in \mathfrak{A}_{0}, s_{i} \neq s_{\overline{1}} .
$$

The condition (16) says that we add anchors living on a new vertical line in the index space, and, for condition (17), that line is mapped onto a new line in the parametric space.

We now extend Lemma 3.2 to the case when new anchors are inserted on a vertical line whose image is already present in the parametric space, that is, we treat the case of knot repetition in the vertical direction. In view of the counter-example shown in Figure 2, this operation may not be always possible. Indeed, the same argument as the one used in proof of Lemma 3.2 can be applied, but under restrictive assumptions on the position of new anchors. 
Lemma 3.4. Consider a linearly independent T-mesh $\mathfrak{M}_{0}$, and a T-mesh $\mathfrak{M}_{1}$ obtained by adding anchors (to $\mathfrak{M}_{0}$ ) with the property that there exists a unique $\overline{\mathrm{j}} \in \mathcal{I}_{\mathfrak{j}}$ such that

$$
\mathfrak{A}_{1} \backslash \mathfrak{A}_{0}=\left\{(i, \overline{\mathrm{j}}), i \in \mathcal{I}_{\mathfrak{i}}\right\} \cap \mathfrak{A}_{1} .
$$

Moreover, let

$$
r_{\overline{\mathrm{j}}, 0}=\max _{i} \#\left\{j:(i, j) \in \mathfrak{A}_{0}, t_{j}=t_{\overline{\mathrm{J}}}\right\} .
$$

If it holds:

$$
\forall i \in\left\{i:(i, \overline{\mathrm{J}}) \in \mathfrak{A}_{1} \backslash \mathfrak{A}_{0}\right\}, \quad \#\left\{j:(i, j) \in \mathfrak{A}_{1}: t_{j}=t_{\overline{\mathrm{J}}}\right\}=r_{\overline{\mathrm{j}}, 0}+1,
$$

then the functions $\left\{B_{1}^{(i, \bar{\jmath})},(i, \overline{\mathrm{J}}) \in \mathfrak{A}_{1} \backslash \mathfrak{A}_{0}\right\}$ are linearly independent, and the following sum is direct

$$
S_{0} \oplus \operatorname{span}\left\{B_{1}^{(i, \bar{\jmath})}(s, t),(i, \overline{\mathrm{J}}) \in \mathfrak{A}_{1} \backslash \mathfrak{A}_{0}\right\} .
$$

Remark 3.5. Note that $r_{\overline{\mathrm{j}}, 0}$ is the maximum number of repetitions in the vertical direction that may occur at $t=t_{\overline{\mathrm{J}}}$. The assumption (19) says that we can add anchors only at locations where the maximum number of vertical knot repetition occurs. See Figure 4 for clarifying drawings. Observe that Lemma 3.2 is in fact a special case of Lemma 3.4, with $r_{\overline{\mathrm{J}}, 0}=0$.

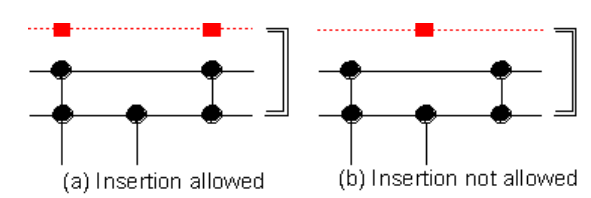

Figure 4: New anchors are designated by rectangles. Dotted lines denote newly added lines on T-mesh in an index/parametric space. Parallel brackets denote repeated lines as in Figure 1. Figures above represent part of index/parametric spaces, and show when the insertion of new anchors is or is not allowed.

Proof of Lemma 3.4. The proof is similar to that of Lemma 3.2. Particularly, if we look at equation (15), the left hand side $\Phi_{\mathfrak{s}}^{(i, \bar{\jmath})}\left(\sum_{(i, j) \in \mathfrak{A}_{0}} \alpha^{(i, j)} B_{0}^{(i, j)}(s, t)\right)$ is $C^{3-r_{\overline{\mathrm{J}}, 0}}$ at $t=t_{\overline{\mathrm{J}}}$ and $N_{\mathrm{t}, 1}^{(i, \overline{\mathrm{J}}}(t)$, appearing at the right hand side of the equation is not $C^{3-r_{\mathrm{J}}, 0}$, but only $C^{2-r_{\mathrm{J}, 0}}$, at the point.

Remark 3.6. By exchanging horizontal with vertical lines in the statement of Lemma 3.4, an equivalent result holds true for the insertion of anchors on a vertical line $i=\overline{1}$ with property similar to that of Lemma 3.4.

Several applications of Lemmas 3.2 and 3.4 and Remarks 3.3 and 3.6, on a sequence of refined T-meshes, lead to the following result.

Theorem 3.7. Let the T-meshes $\mathfrak{M}_{0}, \ldots, \mathfrak{M}_{\ell}$ be given. Assume $\mathfrak{M}_{0}$ is linearly independent, and $\mathfrak{M}_{k}, k=1, \ldots, \ell$ are obtained as sequential refinements by the anchor insertion as described in Lemma 3.4 and Remark 3.6 (or Lemma 3.2 and Remark 3.3). Then $\mathfrak{M}_{\ell}$ is linearly independent. 
Proof. It follows from Lemmas 3.2 and 3.4 and Remarks 3.3 and 3.6 that $\left\{B_{0}^{(i, j)}(s, t)\right\}_{(i, j) \in \mathfrak{A}_{0}}$ and $\left\{B_{1}^{(i, j)}(s, t)\right\}_{(i, j) \in \mathfrak{A}_{1} \backslash \mathfrak{A}_{0}}$ are linearly independent. By Proposition 3.1, the T-mesh $\mathfrak{M}_{1}$ is linearly independent. Repeating this process as $k<\ell$, we obtain that $\left\{B_{k}^{(i, j)}(s, t)\right\}_{(i, j) \in \mathfrak{A}_{k}}$ and $\left\{B_{k+1}^{(i, j)}(s, t)\right\}_{(i, j) \in \mathfrak{A}_{k+1} \backslash \mathfrak{A} \mathfrak{A}_{k}}$ are linearly independent, therefore $\mathfrak{M}_{k+1}$ is linearly independent.

\section{Examples of linearly independent T-splines}

In this section, we use Theorem 3.7 to prove the linear independence after possibly several refinement steps. For clarity, we will adopt a different style in graphically representing these refinements. More precisely, we draw the underlying T-mesh $\mathfrak{M}_{0}$ in the parametric space (and we choose it to be tensor product, for simplicity) and we emphasize the insertion of (images of) new anchors, possibly repeated. Repetition is represented by close anchors with various shapes in the drawing (see Figure 5).

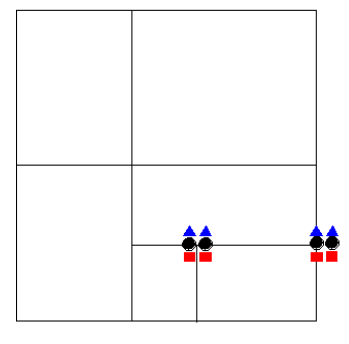

(a) Parametric space

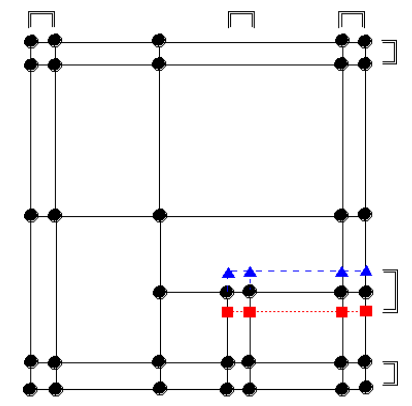

(b) Index/parametric space

Figure 5: New anchors are represented sequentially by rectangles and triangles. Dotted/dashed lines denote newly added lines on T-mesh in the index/parametric space. Parallel brackets in (b) denote repeated lines as in Figure 1.

Moreover, we will also simplify our graphical notation according to Figure 6: i) the knot repetition on newly added lines is often incorporated in a single step, e.g., we draw Figure 6 (b) instead of Figure 6(a); ii) when no confusion occurs or when inserted knots can be either repeated or not without affecting the linear independence, we will also accept the reduced representation of Figure 6(c).

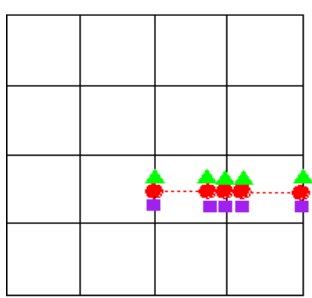

(a)

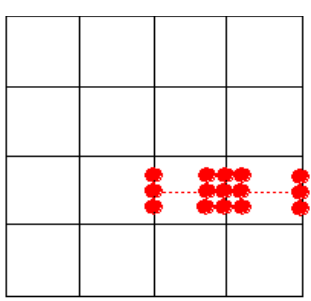

(b)

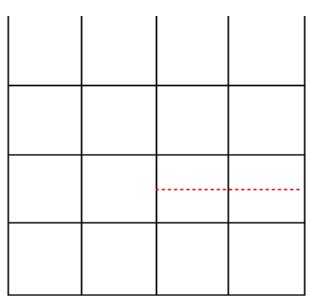

(c)

Figure 6: A T-mesh in the parametric space, represented in three different ways. (a) New anchors are designated by circles, rectangles and triangles. (b) New anchors are identified with circles. (c) A newly added line is denoted by a dotted line on T-mesh in the parametric space. 
We present examples of linearly independent T-meshes of practical interest. In each of these examples, a detailed explanation is provided.

\subsection{Simple T-meshes}

The first two examples are illustrated in Figure 7. We start with a uniform T-mesh $\mathfrak{M}_{0}$ presented in the leftmost column of Figure 7 . At the first step of the refinements, we

(a)

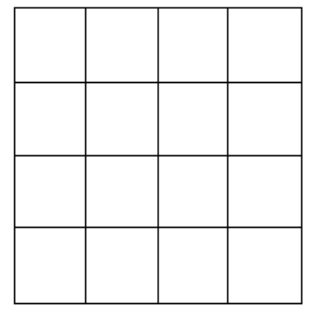

(b)

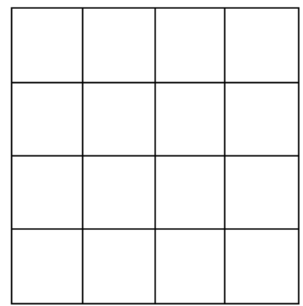

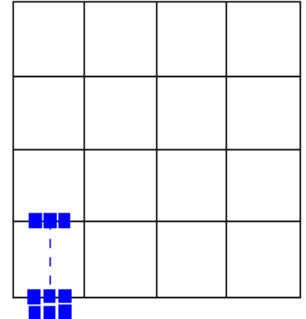

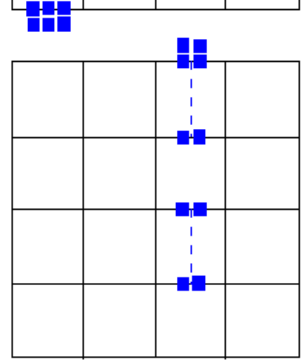

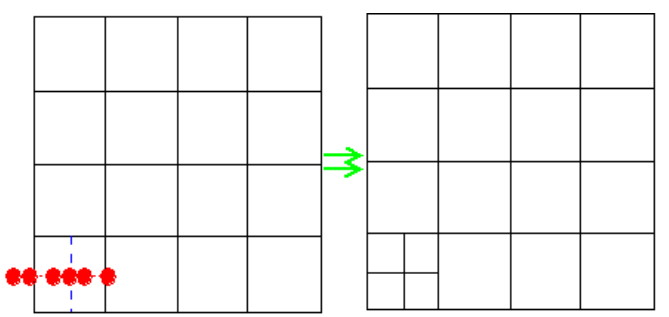

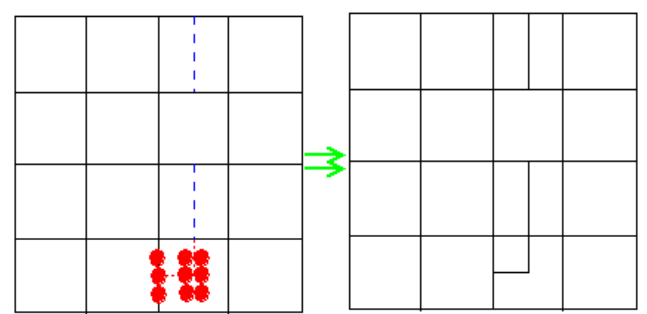

Figure 7: Sequence of T-meshes in parametric space. Newly added lines are denoted by dotted/dashed lines. New anchors are designated by rectangles/circles. The corresponding T-splines to newly added anchors have various continuity order across the new line, which is identified with $C^{3-r}$ where $r$ is the number of repeated anchors in $s$ or $t$ direction.

add rectangular anchors to the T-mesh $\mathfrak{M}_{0}$. Those additions result in a T-mesh $\mathfrak{M}_{1}$. Then Lemma 3.4 ensures that T-mesh $\mathfrak{M}_{1}$ is linearly independent. In next refinement, we insert circular anchors to the T-mesh $\mathfrak{M}_{1}$, which gives a new T-mesh $\mathfrak{M}_{2}$ shown in the rightmost column of Figure 7 . Then, by Theorem 3.7, the T-mesh $\mathfrak{M}_{2}$ is linearly independent.

In next examples, the starting T-meshes $\mathfrak{M}_{0}$ displayed in the leftmost column of Figure 8 are different from uniform T-meshes. Thanks to the example illustrated in Figure 7(a), those T-meshes $\mathfrak{M}_{0}$ are linearly independent. We add circular anchors by the local refinement algorithm, which yields a new T-mesh $\mathfrak{M}_{1}$. Observe that also vertical edges are added, which is allowed by Theorem 3.7. Our result says that the T-mesh $\mathfrak{M}_{1}$ is linearly independent.

\subsection{Hierarchical T-meshes}

We are interested in the linear independence of hierarchical T-meshes. We also begin with uniform T-meshes $\mathfrak{M}_{0}$. With a coarser T-mesh, the local refinement at each step generates a finer hierarchical T-meshes, as illustrated in Figure 9. It is easy to see that newly added lines, designated by dotted/dashed lines in Figure 9, fit into the framework of Theorem 3.7. Then Theorem 3.7 implies that the corresponding hierarchical T-meshes $\mathfrak{M}_{k}$ are linearly independent. Note that repetitions are allowed on the newly inserted lines under the assumptions of Lemma 3.4. 
(a)

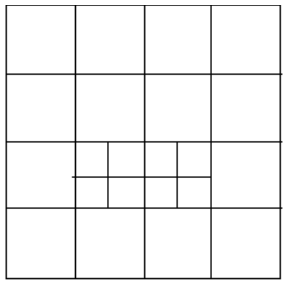

(b)

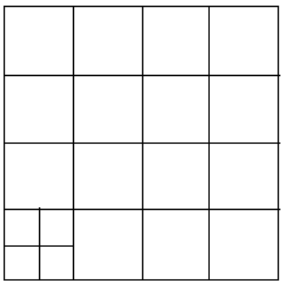

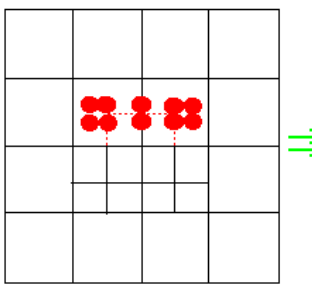
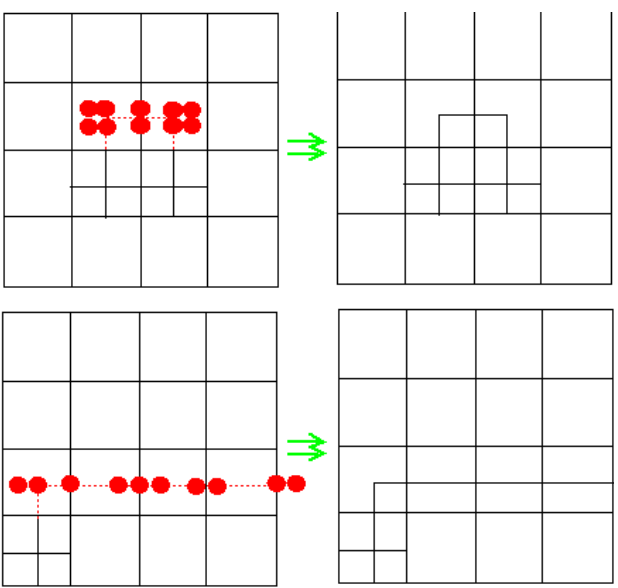

Figure 8: Sequence of T-meshes in parametric space. Newly added lines are denoted by dotted lines. New anchors are designated by circles.

(a)

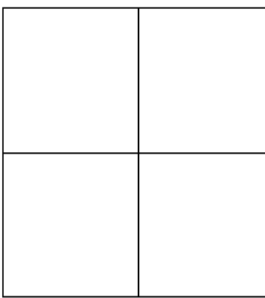

(b)
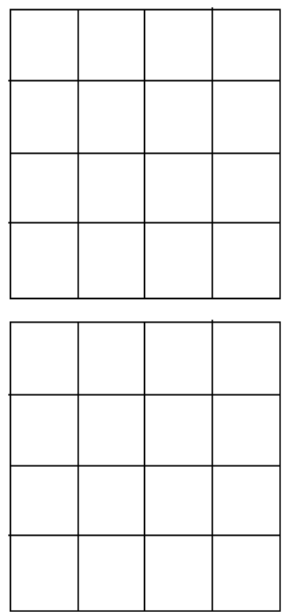
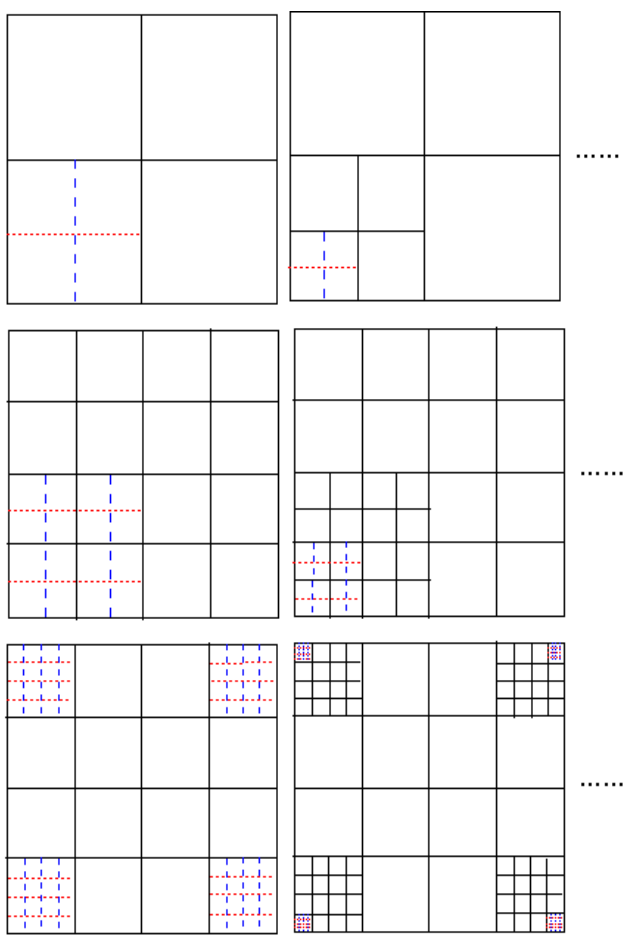

Figure 9: Sequences of hierarchical T-meshes in parametric space. Dotted/dashed lines denote newly added lines. 


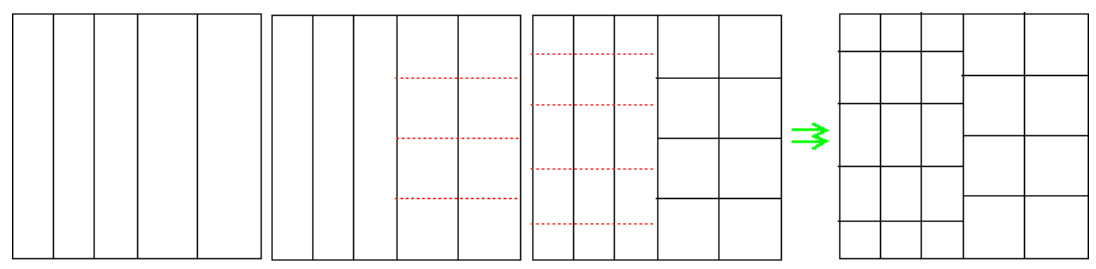

(a) Sequence of nonmatching T-meshes
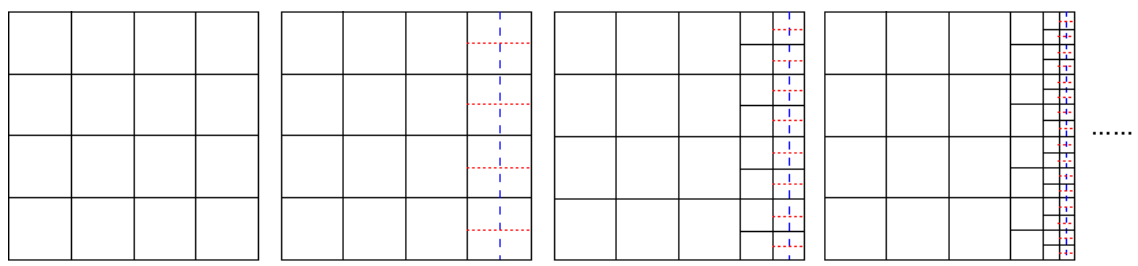

(b) Sequence of T-meshes associated with geometric refinement toward a boundary layer

Figure 10: Dotted/dashed lines denote newly added lines on T-meshes in the parametric spaces.

\subsection{Nonmatching T-mesh and geometrically refined T-meshes on a boundary layer}

Attention is paid to a nonmatching T-mesh and the ones associated with geometric refinement toward a boundary layer as shown in Figure 10. We here discuss the linear independence of those special T-meshes. The first example involves a nonmatching T-mesh presented in the rightmost of Figure 10(a). Starting with B-spline discretization $\mathfrak{M}_{0}$ shown in the left-upmost of Figure 10(a), seven legal dotted lines are added to the T-mesh $\mathfrak{M}_{0}$. Those additions give a new T-mesh $\mathfrak{M}_{7}$. In the end, the nonmatching T-mesh $\mathfrak{M}_{7}$ is linearly independent.

In the second example, a uniform T-mesh $\mathfrak{M}_{0}$ continues to be refined on a boundary layer as shown in Figure 10(b). At each step, legal dotted/dashed lines are added by the local refinement algorithm. It follows from Theorem 3.7 that the resulting geometrically refined T-meshes on the boundary layer are linearly independent.

\subsection{More particular T-meshes}

T-meshes that are referred as a worst case scenario in [12], are presented in Figure 11(a). T-mesh $\mathfrak{M}_{0}$, similar as in [12], is obtained by a tensor product of two global knot vectors $\Xi_{\mathfrak{s}}=$ $\{0,0,0,0,1 / 2,1 / 2,1 / 2,1,1,1,1\}$ and $\Xi_{\mathfrak{t}}=\{0,0,0,0,1 / 2,1 / 2,1 / 2,1,1,1,1\}$, as shown in the left-upmost of Figure 11(a). T-meshes are refined by subdividing patches containing the diagonal from $(0,0)$ to $(1,1)$ into four parts and adding additional anchors (knots) according to the local refinement algorithm (see Figure 11(a)). Figure 11(b-c) shows that T-mesh at each level can be constructed from the coarser T-mesh by several additions of new legal dotted/dashed lines that fall into the framework of Theorem 3.7. Therefore, at each level, the resulting T-meshes are linearly independent.

\section{Concluding remarks}

In this paper, we have proved the linear independence of bi-cubic T-splines on practically interesting T-meshes. Our analysis can be extended to the linear independence of the 

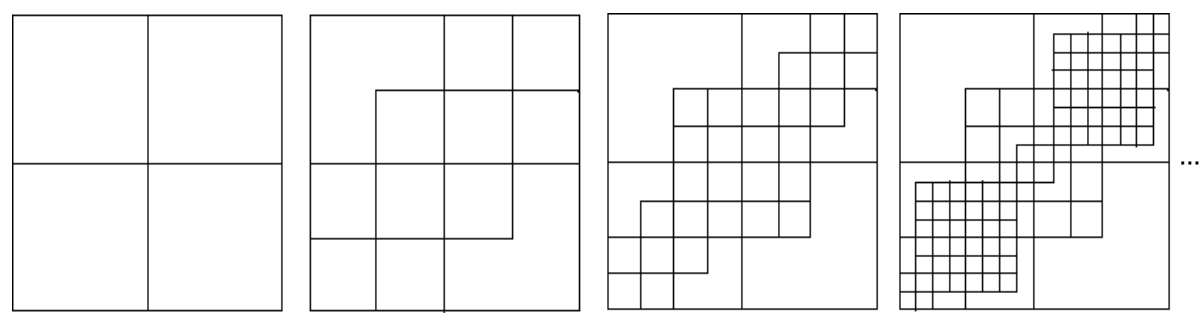

(a) Sequence of T-meshes in parametric space.
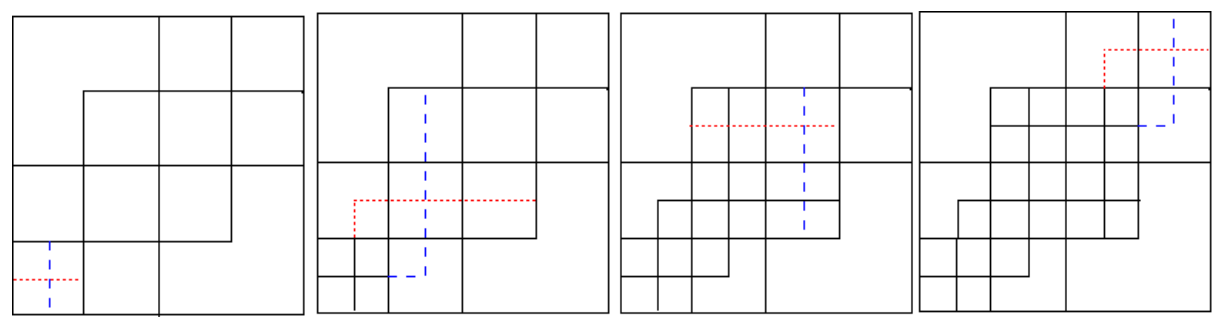

(b) T-mesh at level 3.
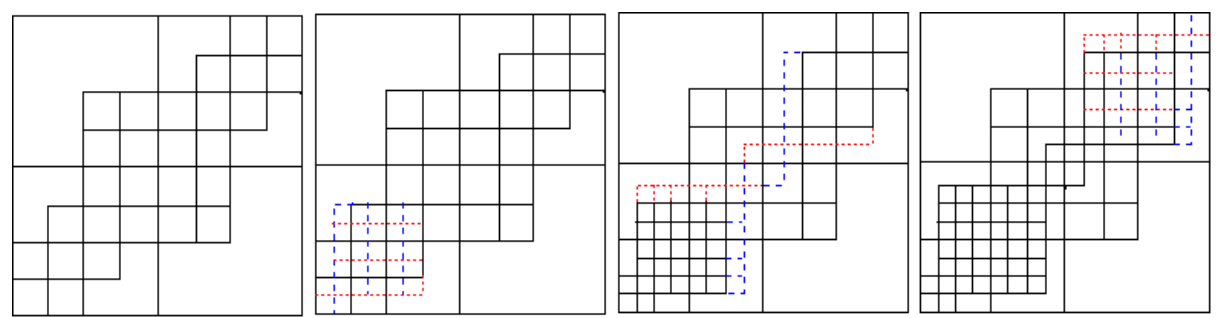

(c) T-mesh at level 4 .

Figure 11: A worst case scenario presented in [12]. Newly added lines are denoted by dotted/dashed lines. 
resulting T-spline blending functions of any odd degree $p$ on all the T-meshes that we have considered in this paper. Our argument covers T-meshes that can be obtained from a coarse T-mesh, which is known to be linearly independent (e.g., a tensor product T-mesh), by a sequence of refinements such that, at each step, only aligned anchors are inserted. It is clear that this is a very particular kind of refinement. Nevertheless we have shown that we can analyze this way the practically interesting T-meshes that have been considered in literature of T-spline based isogeometric analysis. The result we have proposed is only a first step toward the study of general T-meshes, and gives some theoretical grounding to this topic. The complete characterization of linear independence of T-meshes is still open, as other fundamental issues like approximation properties of T-spline spaces.

\section{Acknowledgments}

The authors are deeply indebted to Prof. T.J.R. Hughes, University of Texas at Austin, for pointing out to us the problem partially treated here and for many fruitful discussions. Furthermore, the authors thank Mike Scott, University of Texas at Austin, for interesting comments and insights on this paper. The authors were partially supported by the European Research Council through the FP7 Ideas Starting Grant 205004: GeoPDEs - Innovative compatible discretization techniques for Partial Differential Equations. This support is gratefully acknowledged.

\section{References}

[1] Auricchio, F., Beirão da Veiga, L., Buffa, A., Lovadina, C., Reali, A., Sangalli, G., 2007. A fully "locking-free" isogeometric approach for plane linear elasticity problems: a stream function formulation. Comput. Methods Appl. Mech. Engrg. 197 (1-4), 160172.

[2] Bazilevs, Y., Beirão da Veiga, L., Cottrell, J. A., Hughes, T. J. R., Sangalli, G., 2006. Isogeometric analysis: approximation, stability and error estimates for $h$-refined meshes. Math. Models Methods Appl. Sci. 16 (7), 1031-1090.

[3] Bazilevs, Y., Calo, V., Cottrell, J., Evans, J., Hughes, T., Lipton, S., Scott, M., Sederberg, T., 2009. Isogeometric analysis using T-splines. Comput. Methods Appl. Mech. Engrg., doi:10.1016/j.cma.2009.02.036.

[4] Bazilevs, Y., Calo, V., Zhang, Y., Hughes, T., 2006. Isogeometric fluid-structure interaction analysis with applications to arterial blood flow. Computational Mechanics 38, 310-322.

[5] Bazilevs, Y., Calo, V. M., Cottrell, J. A., Hughes, T. J. R., Reali, A., Scovazzi, G., 2007. Variational multiscale residual-based turbulence modeling for large eddy simulation of incompressible flows. Comput. Methods Appl. Mech. Engrg. 197 (1-4), 173-201.

[6] Bazilevs, Y., Calo, V. M., Hughes, T. J. R., Zhang, Y., 2008. Isogeometric fluid-structure interaction: theory, algorithms, and computations. Comput. Mech. 43 (1), 3-37. 
[7] Bazilevs, Y., Hughes, T., 2008. Nurbs-based isogeometric analysis for the computation of flows about rotating components. Computational Mechanics 43, 143-150.

[8] Beirão da Veiga, L., Buffa, A., Sangalli, G., Rivas, J., 2009. Some estimates for $h-p-k$ refinements in isogeometric analysis. Tech. rep., IMATI-CNR, Pavia.

[9] Buffa, A., Sangalli, G., Vazquez, R., 2009. Isogeometric analysis in electromagnetics: B-splines approximation. Tech. Rep. 7PV09/6/0, IMATI-CNR.

[10] Cottrell, J., Hughes, T., Reali, A., 2007. Studies of refinement and continuity in isogemetric analysis. Computer Methods in Applied Mechanics and Engineering 196, 41604183.

[11] Cottrell, J. A., Reali, A., Bazilevs, Y., Hughes, T. J. R., 2006. Isogeometric analysis of structural vibrations. Comput. Methods Appl. Mech. Engrg. 195 (41-43), 5257-5296.

[12] Dörfel, M., Jüttler, B., Simeon, B., 2008. Adaptive isogeometric analysis by local $h$-refinement with t-splines. Comput. Methods Appl. Mech. Engrg., doi:10.1016/j.cma.2008.07012.

[13] Elguedj, T., Bazilevs, Y., Calo, V., Hughes, T., 2008. $\bar{B}$ and $\bar{F}$ projection methods for nealy incompressible linear and non-linear elasticity and plasticity using higher-order nurbs elements. Computer Methods in Applied Mechanics and Engineering 197, 27322762 .

[14] Evans, J., Bazilevs, Y., Babuška, I., Hughes, T., 2009. n-width, sup-infs, and optimality ratios for the $k$-version of the isogeometic finite element method. Computer Methods in Applied Mechanics and Engineering 198, 1726-1741.

[15] Gómez, H., Calo, V. M., Bazilevs, Y., Hughes, T. J. R., 2008. Isogeometric analysis of the Cahn-Hilliard phase-field model. Comput. Methods Appl. Mech. Engrg. 197 (49-50), 4333-4352.

[16] Hughes, T. J. R., Cottrell, J. A., Bazilevs, Y., 2005. Isogeometric analysis: CAD, finite elements, NURBS, exact geometry and mesh refinement. Comput. Methods Appl. Mech. Engrg. 194 (39-41), 4135-4195.

[17] Schumaker, L. L., 2007. Spline functions: basic theory, 3rd Edition. Cambridge Mathematical Library. Cambridge University Press, Cambridge.

[18] Sederberg, T., Cardon, D., Finnigan, G., North, N., Zheng, J., Lyche, T., 2004. T-spline simplication and local refinement. ACM Transactions on Graphics 23 (3), 276-283.

[19] Sederberg, T., Zheng, J., Bakenov, A., Nasri, A., 2003. T-splines and T-NURCCSs. ACM Transactions on Graphics 22 (3), 477-484.

[20] Wall, W. A., Frenzel, M. A., Cyron, C., 2008. Isogeometric structural shape optimization. Comput. Methods Appl. Mech. Engrg. 197 (33-40), 2976-2988. 
[21] Zhang, Y., Bazilevs, Y., Goswami, S., Bajaj, C. L., Hughes, T. J. R., 2007. Patientspecific vascular NURBS modeling for isogeometric analysis of blood flow. Comput. Methods Appl. Mech. Engrg. 196 (29-30), 2943-2959. 